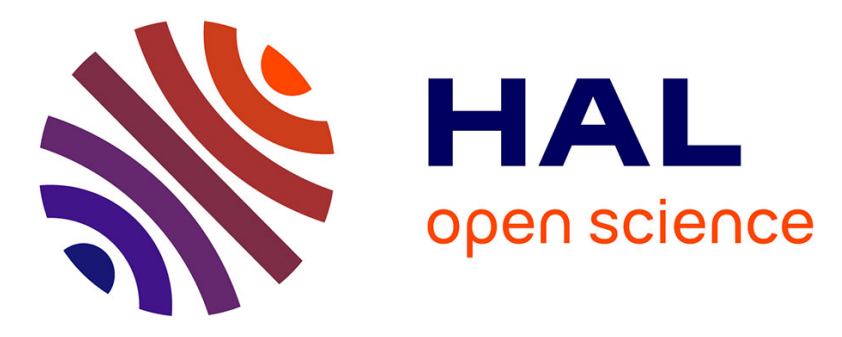

\title{
Skin lesion imaging with line-field confocal optical coherence tomography
}

Maxime Cazalas, Olivier Levecq, Hicham Azimani, David Siret, Anaïs Barut, Mariano Suppa, Véronique del Marmol, Josep Malvehy, Élisa Cinotti, Pietro Rubegni, et al.

\section{To cite this version:}

Maxime Cazalas, Olivier Levecq, Hicham Azimani, David Siret, Anaïs Barut, et al.. Skin lesion imaging with line-field confocal optical coherence tomography. SPIE's International Symposium on Biomedical Optics (BiOS), Feb 2019, San-Francisco, United States. 10.1117/12.2508484 . hal02197166

\section{HAL Id: hal-02197166 \\ https://hal-iogs.archives-ouvertes.fr/hal-02197166}

Submitted on 30 Jul 2019

HAL is a multi-disciplinary open access archive for the deposit and dissemination of scientific research documents, whether they are published or not. The documents may come from teaching and research institutions in France or abroad, or from public or private research centers.
L'archive ouverte pluridisciplinaire HAL, est destinée au dépôt et à la diffusion de documents scientifiques de niveau recherche, publiés ou non, émanant des établissements d'enseignement et de recherche français ou étrangers, des laboratoires publics ou privés. 


\title{
Skin lesion imaging with line-field confocal optical coherence tomography
}

\author{
Maxime Cazalas $^{\mathrm{a}}$, Olivier Levecq ${ }^{\mathrm{a}}$, Hicham Azimani $^{\mathrm{a}}$, David Siret $^{\mathrm{a}}$, Anaïs Barut ${ }^{\mathrm{a}}$, Mariano Suppa ${ }^{\mathrm{b}}$, \\ Véronique del Marmol ${ }^{\mathrm{b}}$, Josep Malvehy ${ }^{\mathrm{c}}$, Elisa Cinotti ${ }^{\mathrm{d}}$, Pietro Rubegni ${ }^{\mathrm{d}}$, Jean-Luc Perrot ${ }^{\mathrm{e}}$, \\ Arnaud Dubois ${ }^{\mathrm{f}}$ \\ ${ }^{a}$ DAMAE Medical, 28 rue de Turbigo, 75003 Paris, France; ${ }^{b}$ Department of Dermatology, Hôpital \\ Erasme, Université Libre de Bruxelles, Brussels, Belgium; ' University Hospital Clinic of Barcelona, \\ Barcelona, Spain; ${ }^{\mathrm{d} D e p a r t m e n t}$ of Medical, Surgical and Neurological Sciences, Dermatology Unit, \\ University of Siena, Siena, Italy; ' University hospital of Saint-Etienne, 42100 Saint-Etienne, France; \\ ${ }^{\mathrm{f}}$ Laboratoire Charles Fabry, Institut d'Optique Graduate School, Univ. Paris-Saclay, 91127 Palaiseau \\ Cedex, France
}

\begin{abstract}
An improved optical coherence tomography (OCT) technique called line-field confocal OCT (LC-OCT) has been developed for high-resolution skin imaging. Combining the principles of time-domain OCT and confocal microscopy with line illumination and detection, LC-OCT acquires multiple A-scans in parallel with dynamic focusing. With a quasi isotropic resolution of $\sim 1 \mu \mathrm{m}$, the LC-OCT images reveal a comprehensive structural mapping of skin, in vivo, at the cellular level down to a depth of $\sim 500 \mu \mathrm{m}$. LC-OCT images of various skin lesions, including carcinomas and melanomas, are found to well correlate with histopathological images. LC-OCT could significantly improve clinical diagnostic accuracy, while reducing the number of biopsies of benign lesions.
\end{abstract}

Keywords: Optical coherence tomography, three-dimensional microscopy, medical imaging, dermatology

\section{INTRODUCTION}

Skin cancer is the most common form of all human cancers ${ }^{1,2}$. Recent studies show the number of skin cancer cases growing at an alarming rate $^{3}$. In addition to causing illness and death, skin cancer is a huge economic burden. Early detection is likely the most promising way to reduce morbidity and mortality. The standard of care procedure for the detection of skin cancer involves visual examination of the superficial structures of the skin using a dermatoscope ${ }^{4-6}$. The clinical and dermoscopic evaluation of skin lesions is then followed by a biopsy, which is a surgical removal of a tissue sample in order to examine it at the cellular-level using an optical microscope. In this procedure, nearly $60 \%$ of all skin biopsies result in benign diagnoses ${ }^{2}$. However, approximately $20 \%$ of skin cancers, including roughly a third of all melanomas, are missed ${ }^{3}$. Given this challenge, improved diagnostic modalities using noninvasive imaging techniques have been developed to provide earlier, more accurate detection of suspicious lesions, thereby decreasing the false positive rates of dermoscopy and thereby the rate of unnecessary biopsies ${ }^{7}$. The clinically available techniques capable of in vivo skin imaging with the highest spatial resolution are reflectance confocal microscopy, nonlinear optical microscopy, and optical coherence tomography, 8,9

Reflectance confocal microscopy (RCM) is an optical imaging technique that allows the analysis of the skin with a nearly histological resolution $(\sim 1 \mu \mathrm{m})$, i.e. at a cellular level ${ }^{10-13}$. Similar to dermoscopy images, real-time images obtained by RCM are oriented horizontal to the skin surface (en face sections). RCM has been applied in the clinical arena for the diagnosis of melanocytic and nonmelanocytic lesions where it has been proven to increase the diagnostic accuracy when coupled with dermoscopy ${ }^{10,11}$. Beyond its application in skin oncology, RCM can be useful to delineate indications for inflammatory and infectious skin conditions. The main limitation of RCM is its relatively low penetration in skin because of strong light scattering. A penetration depth of $\sim 200 \mu \mathrm{m}$ can be achieved, which is not sufficient to image in the dermis ${ }^{8}$. Also problematic is the fact that RCM sections are oriented perpendicular to conventional histological sections, making them difficult to interpret. 
Nonlinear optical microscopy is a high-resolution imaging modality based on nonlinear interactions of light with biological tissues ${ }^{14}$. Compared with optical coherence tomography (OCT), nonlinear microscopy offers a better spatial resolution, similar to that of RCM. Advances in developing multiphoton excitation microscopes with novel contrast mechanisms, such as second harmonic generation, further allow visualization of skin morphology and function ${ }^{15}$. Key limitations of nonlinear optical microscopy are the orientation of the images (en face sections, like RCM), the small field of view, and the relatively weak penetration in skin $(\sim 200 \mu \mathrm{m})$.

Optical coherence tomography (OCT) is an interferometric imaging modality initially introduced in the clinical field of ophthalmology ${ }^{16}$. The first use of OCT in dermatology was demonstrated in $1997^{17}$ and since several manufacturers of OCT systems have made OCT imaging for dermatology purposes commercially available ${ }^{18}$. Commercially-available OCT systems for dermatology can create in vivo cross-sectional and/or en face images of skin with an axial resolution of 3-5 $\mu \mathrm{m}$ and a lateral resolution of $3-8 \mu \mathrm{m}^{18}$. The penetration depth of OCT is $\sim 1 \mathrm{~mm}$. The possibility to evaluate OCT images in a cross-sectional view (perpendicular to the skin surface) makes them easier to compare with histology sections. Compared with RCM and nonlinear optical microscopy, OCT has a larger lateral field of view, ranging from 2 to $10 \mathrm{~mm}$. The biggest potential of OCT in dermatology has thus far been in the diagnosis, delineation, and treatment of non-melanoma skin cancers, especially basal cell carcinomas. Pigmented lesions, on the other hand, continue to pose great challenges in OCT imaging, mainly because of an insufficient imaging spatial resolution ${ }^{19-22}$.

\section{OPTICAL COHERENCE TOMOGRAPHY}

Two main categories of OCT can be distinguished: time-domain OCT (TD-OCT) and frequency-domain OCT (FD$\mathrm{OCT})^{23}$. In TD-OCT, the sample reflectivity profile as a function of depth is acquired point-by-point by scanning the depth in the sample (A-scan). In FD-OCT, the sample reflectivity profile as a function of depth is acquired without scanning the depth, by measuring the spectrum of the interferometric signal. In both TD-OCT and FD-OCT, a B-scan image is then obtained by lateral scanning of the light beam to acquire several adjacent A-scans.

The axial (depth) resolution in OCT is essentially governed by the temporal coherence of the detected light. Improvement of the axial resolution in OCT has been obtained by the emergence of efficient broadband light sources. Outstanding axial resolutions of $\sim 1 \mu \mathrm{m}$ have been achieved in particular with mode-locked lasers ${ }^{24}$ and more recently with supercontinuum laser sources ${ }^{25,26}$.

FD-OCT is superior to TD-OCT in terms of acquisition rate and detection sensitivity ${ }^{27}$, but presents shortcomings, including a limited lateral resolution ${ }^{23}$. Because all points along the depth range in the sample need to be in focus simultaneously, a depth of focus (DOF) at least equal to the depth range is needed, which limits the beam focusing. Several approaches have been reported to improve the lateral resolution in FD-OCT, including extending the DOF by illuminating the sample with a Bessel beam ${ }^{28-30}$ or by using appropriate phase masks ${ }^{31}$. Computational imaging solutions have also been proposed, including interferometric synthetic aperture microscopy ${ }^{32}$ and digital refocusing ${ }^{33-36}$. An alternative approach consists of combining several B-scan images acquired at different depths over a reduced DOF with higher lateral resolution. This approach has been implemented using the Gabor-based fusion method ${ }^{37}$. It can also be apptied by focusing several light beams at different depths ${ }^{38}$.

Unlike FD-OCT, TD-OCT offers the possibility of adjusting the focus as a function of depth, which makes TD-OCT more appropriate to produce high-resolution images. Dynamic focus tracking in TD-OCT with free-space optics has been reported, but the tracking rate was slow ${ }^{39}$. A microelectromechanical systems (MEMS) mirror was designed for high-speed dynamic focus tracking, but without demonstration of the imaging capability in vivo ${ }^{40}$. Another approach consists of acquiring a sequence of images by gradually shifting the focus onto the sample and then fusing together the in-focus imaging zones ${ }^{24}$. This process results in a trade-off between lateral resolution and image acquisition speed. Another method is to collect multiple foci simultaneously with a multifocus fiber tip array ${ }^{41}$. Despite these advances, however, TD-OCT imaging at high lateral resolution using dynamic focusing remains challenging since a high tracking speed is required. 


\section{LINE-FIELD CONFOCAL OPTICAL COHERENCE TOMOGRAPHY}

We have developed an OCT technique called line-field confocal optical coherence tomography (LC-OCT) to produce high-resolution B-scan images in real time with neither the lateral resolution limitation of FD-OCT nor the speed limitation of dynamically-focused TD-OCT ${ }^{42}$. The LC-OCT technique is based on a two-beam interference microscope with line illumination of the sample using a broadband light source and line detection using a line-scan camera. In LCOCT, multiple A-scans are acquired in parallel. The depth scan can then be slower compared to conventional TD-OCT, without increasing the acquisition time of a whole B-scan image. Since the speed of the depth scan is significantly reduced, dynamic focusing is facilitated. A microscope objective can then be dynamically focused to image with high lateral resolution.

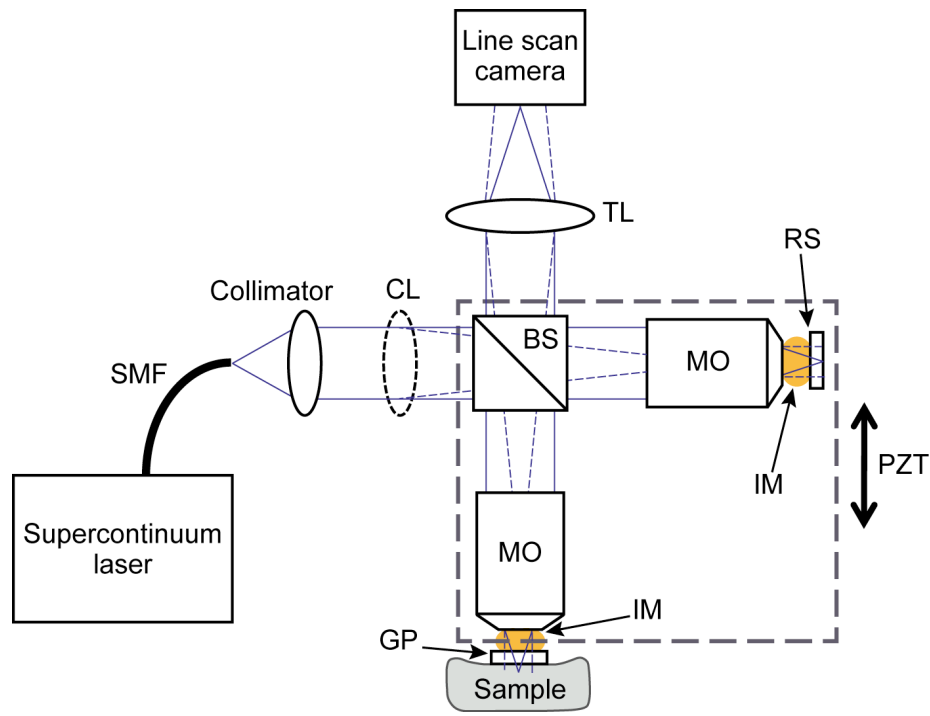

Fig. 1. Schematic of the LC-OCT prototype. TL: tube lens; RS: reference surface; PZT: piezoelectric actuated translation stage; MO : microscope objective; IM: immersion medium; SMF: single mode fiber; BS: beam-splitter; CL: cylindrical lens. GP: glass plate.

LC-OCT is basically a TD-OCT system with a parallel acquisition of multiple A-scans for B-scan imaging without lateral scanning of a light beam as required in conventional OCT. This parallelization facilitates dynamic focusing for Bscan imaging with high lateral resolution at a high (real-time) frame rate. LC-OCT can also be seen as a line-scanning OCM system where the illumination line is scanned axially rather than laterally to acquire B-scan images.

A schematic diagram of the LC-OCT technique is shown in Fig. 1. It consists of a Michelson-type interferometer with a microscope objective placed in each $\mathrm{arm}^{43}$. A supercontinuum laser is used as a source of spatially coherent broadband light. The collimated light beam passes through a cylindrical lens to achieve line illumination in the focal plane of the microscope objectives. The microscope objective in the interferometer reference arm is focused on the external side of a glass plate constituting a low reflectivity $(\sim 4 \%)$ reference surface. An identical glass plate is placed in the sample arm in contact with the biological tissues to image. Immersion microscope objectives are employed with silicon oil as immersion medium placed between the objectives and the glass plates. This medium has an optical refractive index of about 1.4 in the near infrared, similar to the average refractive index of skin tissues. Despite the scan of the sample depth, optical dispersion mismatch in the interferometer arms is minimized to avoid degradation of the axial imaging resolution ${ }^{44}$. This also ensures that the coherence plane (plane of zero optical path difference) and the focus always match $^{44}$ and that the illumination line remains focused in these planes.

The imaging depth within the sample in scanned by displacement of the entire interferometer relative to the sample, as shown in Fig. 1, using a piezoelectric-driven linear stage. A phase-shifting algorithm is applied to extract the fringe envelope from the acquired interferometric data ${ }^{42}$. The calculations are performed with a field-programmable gate array 
(FPGA) to optimize the operation speed. The result of these calculations is a flow of B-scan images that are produced in real-time. The images are displayed in logarithmic scale with auto-adjusted contrast after being appropriately rescaled.

\section{SKIN IMAGING WITH LC-OCT}

\subsection{Imaging protocol}

The evaluation of the LC-OCT device was performed at Saint-Etienne hospital under an institutional review boardapproved protocol ${ }^{45}$. The sterile glass window serves as a mechanical interface between the objective lens of the LCOCT probe and the skin. After adding a drop of paraffin oil between the skin and the glass window, this window is applied to the surface of the patients' skin, gently flattening and stabilizing the area to be imaged. The purpose of the paraffin oil is to provide refractive index matching between the window and the skin, reducing the specular backreflection from both the skin surface and the glass window. After LC-OCT imaging, the lesions were surgically removed according to routine practice protocols. The excised tissues were then processed for histopathological examination. Following chemical fixation, dehydration and clearing, the tissues were embedded in paraffin wax and sectioned in slices using a microtome. The histological sections were then stained using the common Haemotoxylin and Eosin (H\&E) staining system for observation using conventional optical microscopy. Microscope objectives with 20x magnification were employed. This is the same magnification provided by the objectives used in the LC-OCT device.

\subsection{Skin lesions imaging}

LC-OCT has been applied to the imaging of various human skin lesions, in vivo, including Melanocytic lesions and Basal Cell Carcinoma (BCC), at the University hospital of Saint-Etienne, France. Compared to the healthy skin, BCC lesions exhibit lobular structures that appear as dense nest of basal cells, separated from the surrounding stroma by a dark cleft and a bright rim (Fig. 2), as described in previous studies using other OCT devices ${ }^{46,47}$. The lobules can have various shape, size and location (Fig. 3). One advantage of the cross-sectional images produced by LC-OCT, as compared to the en face sections, is that it makes it easier to distinguish between a lobule connected to the epidermis (superficial BCC form) and a lobule separated from the epidermis, which is of crucial importance to select the best therapeutic option for the patient. Another advantage is that the interpretation of the image is straightforward, as it shows a high level of similarity with conventional H\&E histological images (Fig. 3).
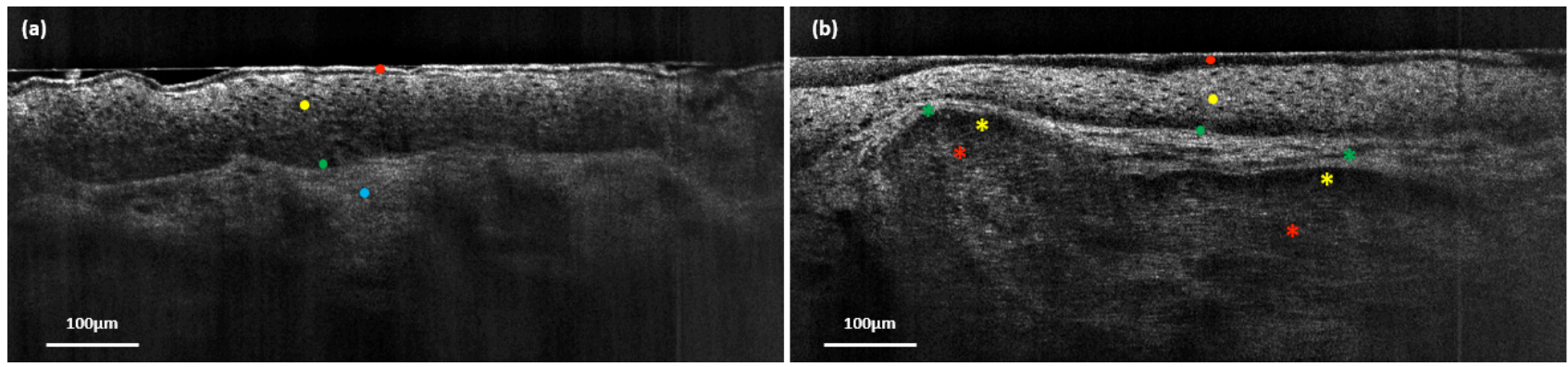

Fig. 2. Comparison between healthy skin and BCC lesion on the nose. (a) image of the nose of a healthy volunteer (22 years old female, phototype 2), red dot: Stratum Corneum, yellow dot: Stratum Spinosum with dark keratinocytes nuclei, green dot: dermal-epidermal junction (DEJ), blue dot: upper dermis. (b) image of a BCC lesion on the nose (79 years old male). red dot: Stratum Corneum, yellow dot: Stratum Spinosum with dark keratinocytes nuclei, green dot: DEJ, red star: dense nest of basal cells (BCC lobule), yellow star: dark cleft at the upper boundary of the BCC lobule, green star: bright rim of stroma surrounding the lobule. 

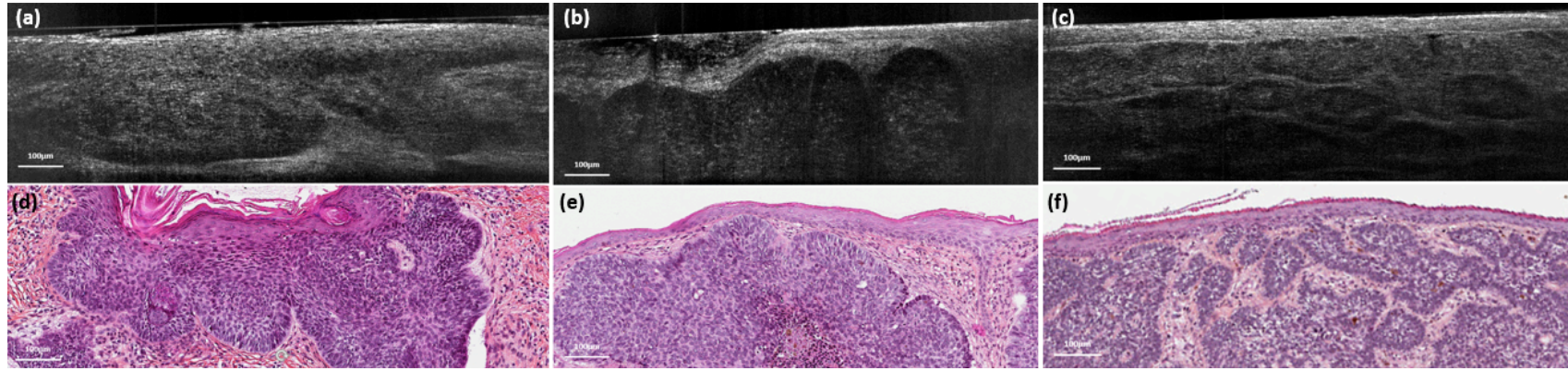

Fig. 3. Similarity between LC-OCT cross-sectional images and conventional H\&E histological images for various shape, size and location of BCC lobules. (a) superficial BCC showing hemispheric lobules appended to the epidermis. (b) nodular BCC with large nodules separated from the epidermis. (c) coalescence of small lobules forming a nodular BCC. A high level of similarity of the shape, size and location of each BCC lobule can be observed between LC-OCT ((a), (b), (c)) and corresponding H\&E images ((d), (e), (f)).

When imaging melanocytic lesions with LC-OCT, most of the histological features that are used to discriminate a benign nevus from a malignant melanoma are visible (Fig. 4). Thanks to the cellular resolution, we can assess architectural disarrangement and detect the presence of atypical melanocytes whose appearance (cytoplasm, nucleus or both) is different from the patient's normal counterpart. Again, the cross-sectional view provided by LC-OCT enable to better reveal a disruption of the DEJ and assess the location of the cellular atypia with respect to the DEJ.
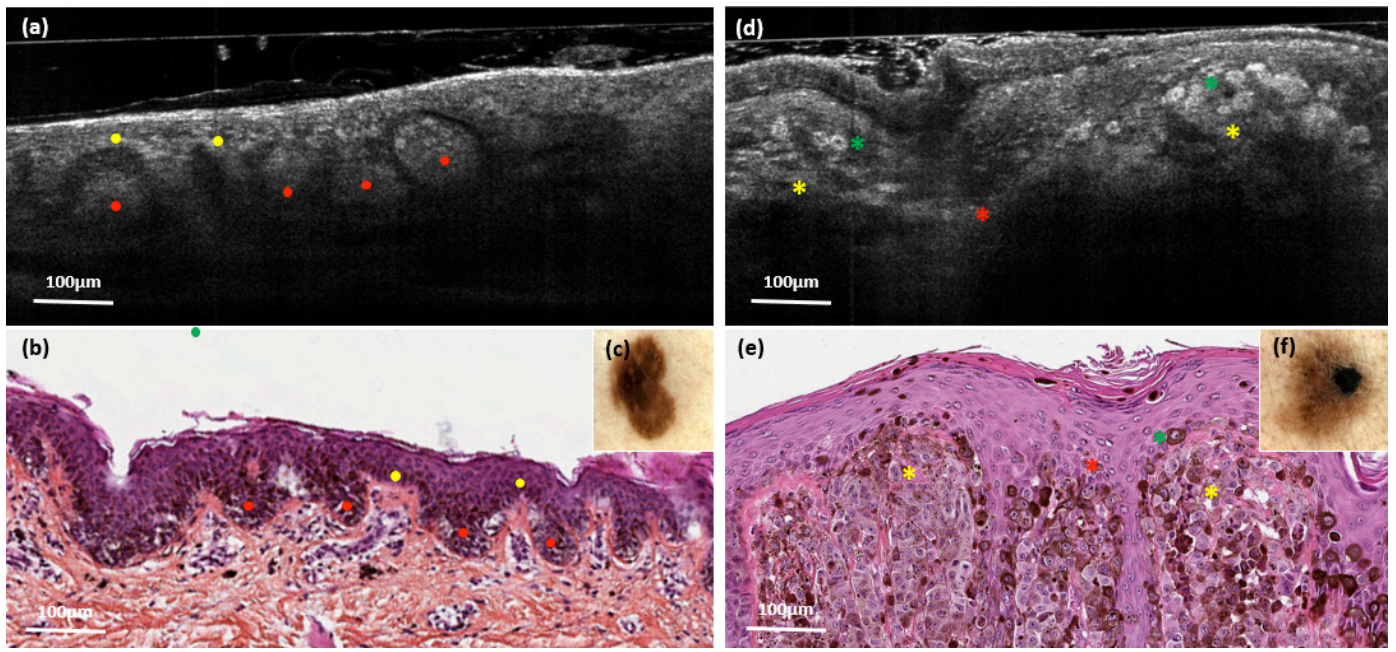

Fig. 4. LC-OCT visible features of melanocytic lesions. (a), (b), (c) benign nevus, resp. LC-OCT, H\&E and dermoscopic images. DEJ is preserved and appears slightly brighter due to melanin pigment distributed along the basal layer (yellow dot), multiple regular clusters of melanocytes appear at the rete ridges and papillae (red dots). (d), (e), (f) superficial spreading Melanoma, resp. LC-OCT, H\&E and dermoscopic images. Pagetoid spread of atypical melanocytes, highly pigmented, with a large ovoid shape and prominent dark nucleus (green star). Dermal nest of atypical melanocytes (yellow star) and partial disruption of the DEJ (red star) are also visible.

\section{CONCLUSION}

An optical technique called Line-field Confocal Optical Coherence Tomography (LC-OCT) has been used for highresolution, non-invasive imaging of human skin in vivo. LC-OCT delivers tomographic images of skin in real-time (10 frames/s) with a quasi isotropic spatial resolution of $\sim 1 \mu \mathrm{m}$, revealing a morphological mapping of tissues at a cellular level, down to a depth of $\sim 500 \mu \mathrm{m}$. LC-OCT has been applied to imaging of various skin lesions, including carcinomas and melanomas. Surgical excisions of the lesions have then been performed followed by tissue processing to realize H\&E-stained histopathological images. The spatial resolution, orientation, and imaging contrast mechanism of the LC- 
OCT images allowed for a good level of similarity with the conventional histopathological images. LC-OCT was able to show most of the histopathological elements that allow for medical diagnosis. With its unprecedented performance, LCOCT will push the limits of in vivo exploration of skin. Using LC-OCT as an adjunct tool in dermatology could help improve clinical diagnostic accuracy, allowing for the early detection of malignant skin lesions and a reduction in the number of surgical excisions of benign lesions.

\section{ACKNOWLEDGEMENTS}

The authors thank the "Ministère de l'Enseignement Supérieur et de la Recherche (MESR)" and the "Banque Publique d'Investissement France (BPI France)" for financial supports.

\section{REFERENCES}

[1] Bickers, D.R. et al., "The burden of skin diseases in 2004: a joint project of the American Academy of Dermatology Association and the Society for Investigative Dermatology," J. Am. Acad Dermatol 55, 490 (2006).

[2] Apalla Z. et al., "Epidemiological trends in skin cancer," Dermatol. Pract. Concept 7, 1-6 (2017).

[3] Mc Carthy M., "US melanoma prevalence has doubled over past 30 years," BMJ 350, h3074 (2015).

[4] Fuller S. R. et al., "Digital dermoscopic monitoring of atypical nevi in patients at risk for melanoma," Dermatol. Surg. 33, 1198 (2007).

[5] Kittler H., Pehamberger H., Wolff K., Binder M., "Diagnostic accuracy of dermoscopy," Lancet Oncol. 3,159 (2002).

[6] Woltsche N., Schwab C., Deinlein T., et al., "Dermoscopy in the era of dermato-oncology: from bed to bench side and retour," Expert Rev. Anticancer Ther. 16, 531-41 (2016).

[7] Glazer A.M., Rigel D.S., Winkelmann R.R., Farberg A.S., "Clinical Diagnosis of Skin Cancer: Enhancing Inspection and Early Recognition," Dermatol. Clin. 35, 409-16 (2017).

[8] Kollias N., Stamatas G.N. "Optical non-invasive approaches to diagnosis of skin diseases," J. Investig. Dermatol. Symp. Proc. 7, 64-75 (2002).

[9] Fink C., Haenssle H.A., "Non-invasive tools for the diagnosis of cutaneous melanoma," Skin Res. Technol. 23, 261-271 (2017).

[10] Pellacani G., Guitera P., Longo C., Avramidis M., Seidenari S., Menzies S., "The impact of in vivo reflectance confocal microscopy for the diagnostic accuracy of melanoma and equivocal melanocytic lesions," J. Invest. Dermatol. 127, 2759-2765 (2007).

[11] Guitera P., Pellacani G., Crotty K.A., Scolyer R.A., Li L.X., Bassoli S., Vinceti M., Rabinovitz H., Longo C., Menzies S.W., "The impact of in vivo reflectance confocal microscopy on the diagnostic accuracy of lentigo maligna and equivocal pigmented and nonpigmented macules of the face," J. Invest. Dermatol. 130, 2080-2091 (2010).

[12] Alarcon I., Carrera C., Palou J., et al., "Impact of in vivo reflectance confocal microscopy on the number needed to treat melanoma in doubtful lesions," Br. J. Dermatol. 170, 802-808 (2014).

[13] Champin J., Perrot J.L., Cinotti E., Labeille B., Douchet C., Parrau G., Cambazard F., Seguin P., Alix T. "In vivo reflectance confocal microscopy to optimize the spaghetti technique for defining surgical margins of lentigo maligna," Dermatol. Surg. Mar. 40, 247-256 (2014).

[14] Zipfel W.R. et al. "Nonlinear magic: multiphoton microscopy in the biosciences," Nat. Biotechnol. 21, 1369-1377 (2003).

[15] Koenig K. et al., "High-resolution multiphoton tomography of human skin with subcellular spatial resolution and picosecond time resolution," J. Biomed. Opt. 8, 432-439 (2003).

[16] Huang D., Swanson E.A., Lin C.P., Schuman J.S., Stinson W.G., Chang W., Hee M.R., Flotte T., Gregory K., Puliafito C.A., Fujimoto J.G., "Optical coherence tomography," Science 254, 1178-1181 (1991).

[17] Welzel J., "Optical coherence tomography of the human skin," J. Am. Acad. Dermatol. 37, 958-963 (1997).

[18] Levine A., Wang K., Markowitz O., "Optical Coherence Tomography in the Diagnosis of Skin Cancer," Dermatol. Clinics 35, 465-488 (2017).

[19] Welzel, J. "Optical Coherence Tomography in Dermatology: A Review," Skin Res. Technol. 7, 1-9 (2001). 
[20] Boone M.A., Norrenberg S., Jemec G.B., Del Marmol V. "Imaging of basal cell carcinoma by high-definition optical coherence tomography: histomorphological correlation. A pilot study," Br. J. Dermatol. 167, 856-864 (2012).

[21] Coleman A.J., Richardson T.J., Orchard G., Uddin A., Choi M.J., Lacy K.E., "Histological Correlates of Optical Coherence Tomography in Non-Melanoma Skin Cancer," Skin Res. Technol. 19, 10-19 (2013).

[22] Ulrich M., Von Braunmuehl T., Kurzen H., Dirschka T., Kellner C., Sattler E., Berking C., Welzel J., Reinhold U., "The Sensitivity and Specificity of Optical Coherence Tomography for the Assisted Diagnosis of Nonpigmented Basal Cell Carcinoma: An Observational Study," Br. J. Dermatol. 173, 428-435 (2015).

[23] Podoleanu A.Gh. "Optical coherence tomography," J. Microscopy 247, 209-219 (2012).

[24] Drexler W., Morgner U., Kärtner F., Pitris C., Boppart S., Li X., Ippen E., Fujimoto J.G., "In vivo ultrahighresolution optical coherence tomography," Opt. Lett. 24, 1221-1223 (1999).

[25] Povazay B., Bizheva K., Unterhuber A., Hermann B., Sattmann H., Fercher A.F., Drexler W, et al. Opt. Lett. 27, 1800-1802 (2002).

[26] Wang Y., Zhao Y., Nelson J.S., Chen Z., Windeler R.S., "Ultrahigh-resolution optical coherence tomography by broadband continuum generation from a photonic crystal fiber," Opt. Lett. 28, 182-184 (2003).

[27] Choma M.A., Sarunic M.V., Yang C., Izatt J.A., "Sensitivity advantage of swept source and Fourier domain optical coherence tomography," Opt. Express 11, 2183-2189 (2003).

[28] Ding Z., Ren H., Zhao Y., Nelson J.S., Chen Z., "High-resolution optical coherence tomography over a large depth range with an axicon lens," Opt. Lett. 27, 243-245 (2002).

[29] Leitgeb R.A., Villiger M., Bachmann A.H., Steinmann L., Lasser T., "Extended focus depth for Fourier domain optical coherence microscopy," Opt. Lett. 31, 2450-2452 (2006).

[30] Lee K.S., Rolland J.P. "Bessel beam spectral-domain high-resolution optical coherence tomography with microoptic axicon providing extended focusing range," Opt. Lett. 33, 1696-1698 (2008).

[31] Mo J., de Groot M., de Boer J.F., "Focus-extension by depth-encoded synthetic aperture in Optical Coherence Tomography," Opt. Express 21, 10048-10061 (2013).

[32] Ralston T.S., Marks D.L., Carney P.S., Boppart S.A., "Interferometric synthetic aperture microscopy," Nat. Phys. 3, 129-134 (2007).

[33] Yu L., Rao B., Zhang J., Su J., Wang Q., Guo S., Chen Z., "Improved lateral resolution in optical coherence tomography by digital focusing using two- dimensional numerical diffraction method," Opt. Express 15, 7634-7641 (2007).

[34] Grebenyuk A., Federici A., Ryabukho V., Dubois A., "Numerically focused full-field swept-source optical coherence microscopy with low spatial coherence illumination," Appl. Opt. 53, 1697-1708 (2014).

[35] D. J. Fechtig, A. Kumar, W. Drexler, and R.A. Leitgeb, "Full range line-field parallel swept source imaging utilizing digital refocusing," J. Mod. Opt. 62, 1801-1807 (2015).

[36] S. Liu, J.A. Mulligan, and S.G. Adie, "Volumetric optical coherence microscopy with a high space-bandwidth-time product enabled by hybrid adaptive optics," Biomed. Opt. Express 9, 3137-3152 (2018).

[37] Rolland J.P., Meemon P., Murali S., Thompson K.P., Lee K.S., "Gabor-based fusion technique for Optical Coherence Microscopy," Opt. Express 18, 3632-3642 (2010).

[38] Holmes J., Hattersley S., "Image blending and speckle noise reduction in multi-beam OCT," in Optical Coherence Tomography and Coherence Domain Optical Methods in Biomedicine XIII, Proc. of SPIE 7168, 71681N (2009).

[39] Schmitt J.M., Lee S.L., Yung K.M., "An optical coherence microscope with enhanced resolving power in thick tissue," Opt. Commun. 142, 203-207 (1997).

[40] Qi B., Himmer P.A., Gordon M.L., Yang V.X.D., Dickensheets D.L., Vitkin I.A., "Dynamic focus control in highspeed optical coherence tomography based on a microelectromechanical mirror," Opt. Comm. 234, 443-448 (2004).

[41] Yang V.X.D., Munce N., Pekar J., Gordon M.L., Lo S., Marcon N.E., Wilson B.C., Vitkin I.A., "Micromachined array tip for multifocus fiber-based optical coherence tomography," Opt. Lett. 29, 1754-1756 (2004).

[42] A. Dubois, O. Levecq, H. Azimani, A. Davis, J. Ogien, D. Siret, A. Barut, "Line-field confocal time-domain optical coherence tomography with dynamic focusing," Opt. Express 26, 33534-33542 (2018).

[43] Dubois, A., Vabre, L., Boccara, A.C., Beaurepaire, E., "High-resolution full-field optical coherence tomography with a Linnik microscope," Appl. Opt. 41, 805-812 (2002).

[44] Dubois A., "Focus defect and dispersion mismatch in full-field optical coherence microscopy," Appl. Opt. 57, 142150 (2017).

[45] Dubois A., Levecq O., Azimani H., Siret D., Barut A., Suppa M., Del Marmol V., Malvehy J., Cinotti E., Perrot J.L., "Line-field confocal optical coherence tomography for high-resolution noninvasive imaging of skin tumors," J. 
Biomed. Opt. 23, 106007 (2018).

[46] Boone M. A. L. M., Suppa M., Pellacani G., Marneffe A., Miyamoto M., Alarcon I., Ruini C., Hofmann-Wellenhof R., Malvehy J., Jemec G. B. E., Del Marmol V., "High-definition optical coherence tomography algorithm for discrimination of basal cell carcinoma from clinical BCC imitators and differentiation between common subtypes", JEADV; 10.1111/jdv.13003 (2015).

[47] Boone M. A. L. M., Norrenberg S., Jemec G. G. et al. "Imaging of basal cell carcinoma by high-definition optical coherence tomography: histomorphological correlation. A pilot study." The British journal of dermatology 167, 856-64 (2012). 